\title{
Atomic Structure of Ultra-Thin WSe2 Films Based on HRTEM Analysis
}

S. Rouvimov, ${ }^{*}$ P. Plachinda, ${ }^{*}$ M. Beekman, ${ }^{* *}$ N. Nguyen, ${ }^{* *}$ D. Johnson, ${ }^{* *}$ P. Moeck, ${ }^{*}$ E. F. Rauch $^{* * *}$

* Department of Physics, Portland State University, Portland, OR 97207-0751

** Department of Chemistry, University of Oregon, Eugene, OR, 97403-1253

${ }^{* * *}$ SIMAP/GPM2 Laboratory, CNRS-Grenoble INP, France

Novel layered thin films that consist of $\mathrm{WSe}_{2}$ (Fig. 1) rotationally disordered sheets have been found to exhibit the lowest ever measured thermal conductivity in a fully-dense solid [1]. It has been suggested that the ultra-low thermal conductivity is achieved by controlling the thermal pathways in this anisotropic material that may be linked to the atomic and electronic structure $[1,2]$. In contrast to bulk crystalline $\mathrm{WSe}_{2}$, High-Resolution Transmission Electron Microscopy (HRTEM) of cross-sectional samples evidenced that the films consist of nm-size ordered domains within a single Se-W-Se layer (hereafter referred to as basal plane units) of disc-like shape which exhibit very little long range registration between the individual $\mathrm{WSe}_{2}$ layers [2]. While the basal plane units were almost perfectly oriented in the direction perpendicular to the substrate, no obvious texture was observed in the azimuthal orientation. Observations in plan-view have not yet been performed for these materials.

The present paper focuses on plan-view HRTEM analysis of the atomic structure of low dimensional (i.e. a few nm thick) layered $\mathrm{WSe}_{2}$ films. Thin $\mathrm{WSe}_{2}$ films have been synthesized by the modulated elemental reactants method [3]. Sequential bilayers of W and Se were deposited in an ultrahigh vacuum chamber on the Si (100) surface and then annealed for 1 hour at elevated temperatures in a $\mathrm{N}_{2}$ atmosphere to form the desired layered structures. The $\mathrm{WSe}_{2}$ films are stable at room temperature.

Due to weak adhesion to the substrate, the films were easily peeled off from the substrate, and then transferred to a standard (amorphous carbon covered) copper grid for electron microscopy analysis. Films consisting of only a few monolayers of $\mathrm{WSe}_{2}$ were also deposited directly on $\mathrm{SiO}_{2} / \mathrm{Si}$ TEM grids followed by thermal annealing and TEM analysis.

The films have been studied by HRTEM either at a FEI Tecnai $\mathrm{G}^{2}$ F20 ST electron microscope (at Portland State University) or at an aberration-corrected FEI Titan 80-300 (at-CAMCOR of the University of Oregon). The effects of the number of $\mathrm{WSe}_{2}$ layers and imaging conditions on the HRTEM contrast and electron diffraction have been also analyzed by multi-slice simulations.

TEM/HREM images and electron diffraction shown in Fig. 2 evidenced that all films consist of textured layers (with basal plane unit dimensions ranging from 2 to $6 \mathrm{~nm}$ ). The majority of basal plane units are oriented with the c-axis-normal to the substrate surface, while the basal plane units are randomly oriented within the a-b plane. Occasionally, basal plane units were observed with the $\mathrm{c}$-axis in the film plane. For very thin films (2-3 WSe 2 stacks), formation of islands was observed, which later merge and form a complete, connected film. To study the structural order and texture of the films, the crystallite orientation mapping has been created on the basis on the analysis of 
Fourier Transforms (computed digital electron diffractograms) of suitable high-resolution images [5]. Further development of these metrologies is anticipated.

\section{References}

[1] C. Chiritescu, D.G. Cahill, N. Nguyen, D. Johnson et.al., Science 315 (2007) 351

[2] S. Kim, J. M. Zuo, N. T. Nguyen and D. C. Johnson et. al., J. Mater. Res. 23 (2008) 1064

[3] S. Moss, M. Noh, K. H. Jeong, D. H. Kim, D. C. Johnson, Chem. Mater. 8 (1996) 1853

[4] A. Enyashin, S. Gemming, and G. Seifert, Eur. Phys. J. Special Topics 149 (2007)103

[5] E. Rauch et al., Microscopy and Microanalysis, 93 (2008) S5
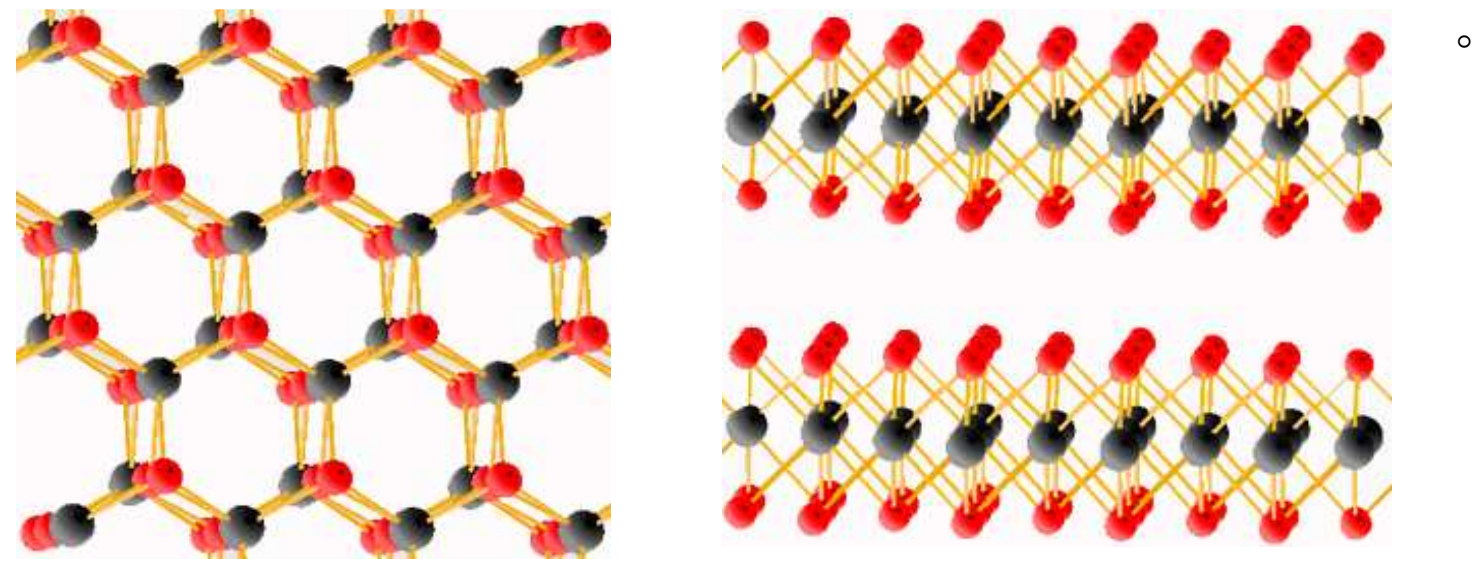

FIG 1: Atomic model for bulk $\mathrm{WSe}_{2}: P 6_{3} / m m c$ polytype in two projections: along c-axis (left) and a-axis (right). Red are Se atoms, grey $-\mathrm{W}$ atoms. $a=0.328 \mathrm{~nm}, b=0.328 \mathrm{~nm}, c=1.296 \mathrm{~nm}, \alpha=$ $90, \beta=90, \gamma=120$
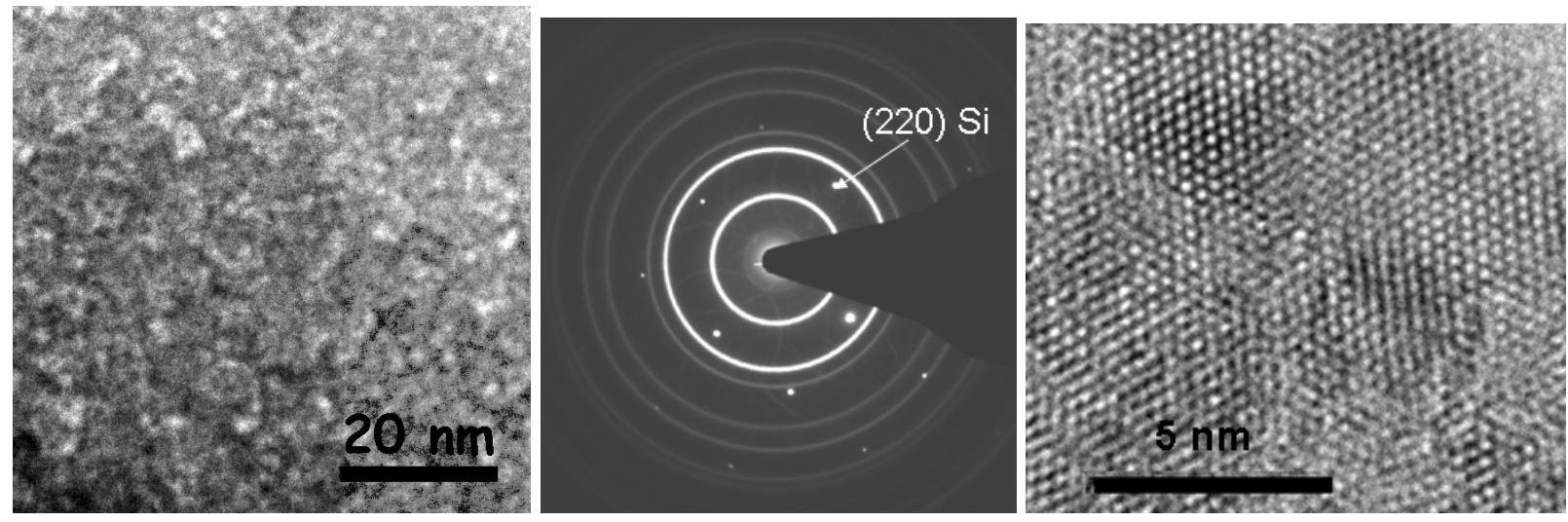

FIG 1: Low magnification bright-field TEM image (a), selected area electron diffraction pattern (b) and HRTEM (c) image of $\mathrm{WSe}_{2}$ sample in plan-view, taken at a FEI Tecnai $\mathrm{G}^{2}$ F20 ST on the edge of a film. 